\title{
One-Family Walking Technicolor in Light of LHC Run-II
}

\author{
Shinya Matsuzaki \\ Department of Physics, Nagoya University, Nagoya 464-8602, Japan. \\ Institute for Advanced Research, Nagoya University, Nagoya 464-8602, Japan.
}

\begin{abstract}
The LHC Higgs can be identified as the technidilaton, a composite scalar, arising as a pseudo Nambu-Goldstone boson for the spontaneous breaking of scale symmetry in walking technicolor. One interesting candidate for the walking technicolor is the QCD with the large number of fermion flavors, involving the one-family model having the eight-fermion flavors. The smallness of the technidilaton mass can be ensured by the generic walking feature, Miransky scaling, and the presence of the "anti-Veneziano limit" characteristic to the large-flavor walking scenario. To tell the standard-model Higgs from the technidilaton, one needs to wait for the precise estimate of the Higgs couplings to the standard model particles, which is expected at the ongoing LHC-Run II. In this talk the technidilaton phenomenology in comparison with the LHC Run-I data is summarized with the special emphasis placed on the presence of the anti-Veneziano limit supporting the lightness of technidilaton. Besides the technidilaton, the walking technicolor predicts the rich particle spectrum such as technipions and technirho mesons, arising as composite particles formed by technifermions. The LHC phenomenology of those technihadrons and the discovery channels are also discussed, which are smoking-guns of the walking technicolor, to be accessible at the LHC-Run II.
\end{abstract}

\section{Introduction}

A Higgs boson with the mass about $125 \mathrm{GeV}$ was discovered at the LHC Run $\mathrm{I} \underline{1}$ and its coupling property has so far been almost consistent with the Higgs boson predicted in the standard model (SM). Yet the dynamical origin of the Higgs, related to the issue left in the SM (such as the naturalness problem) has been uncovered, which is of great importance to be explored at the ongoing LHC Run II.

The dynamical origin of the electroweak symmetry breaking and the Higgs can elegantly be supplied by so-called technicolor $(\mathrm{TC})^{2} \underline{2}, \underline{3}$. However, the technicolor based on the naive-scale up version of QCD (QCD-like technicolor) has severely been disfavored by several inconsistencies with experiments. Most dramatically, it was ruled out by the recent discovery of the Higgs at LHC, because of the absence of the light Higgs at around $125 \mathrm{GeV}$ in the QCD-like technicolor.

In sharp contrast, the walking technicolor (WTC) $\underline{\underline{4}}, \underline{\underline{5}}$ predicts a light composite Higgs, which we call the technidilaton (TD). The TD arises as a pseudo NambuGoldstone (NG) boson for the spontaneous breaking of the approximate scale symmetry of the WTC, triggered by technifermion condensation. Thus its lightness can potentially be ensured by the approximate scale symmetry inherent to the WTC.

One interesting candidate to realize the walking theory is one-family model with 
the number of techniflavors $N_{F}=8 \stackrel{3 \cdot 6}{\underline{n}}$, classified into QCD with the large number of flavors. As it will turn out, actually, the large flavor nature of the walking provides us with a limit where the TD can be regarded as an exactly massless NG boson, analogously to the $\eta^{\prime}$ in the Veneziano limit of QCD. That is what will be called "anti-Veneziano limit".

The LHC signatures of the TD in the one-family model of WTC were stud$\operatorname{ied}^{7} \underline{-13}$. It has been shown $\underline{9}, \underline{12}, 13$ that when the walking theory has the number of technicolor $N_{C}=4$, the coupling property of the $125 \mathrm{GeV}$ TD is consistent with the LHC Higgs at the almost same level as the SM Higgs.

This talk summarizes the $125 \mathrm{GeV}$ TD phenomenology at the LHC Run I and discusses future prospect in the Run II. The emphasis is also placed on several supports on the theoretical ground that the TD can indeed be as light as the 125 $\mathrm{GeV}$ boson due to the intrinsic feature of large flavor WTC. Besides the TD, the walking technicolor predicts the rich particle spectrum such as technipions and technirho mesons, arising as composite particles formed by technifermions, The LHC phenomenology of those technihadrons and the discovery channels are also discussed, which are smoking-guns of the walking technicolor, to be accessible at the LHC-Run II.

\section{Characteristic Features of Walking Technicolor}

In Fig. 1 a schematic view of the WTC is depicted in terms of the gauge coupling $\alpha$ (left panel) and its beta function $\beta(\alpha)$ (right panel). The walking, almost nonrunning region is literally defined as the domain where the running behavior of $\alpha$ looks like almost constant in scale (or the beta function $\beta$ looks almost zero in terms of the beta function $\beta$ ). The presence of walking region implies a pseudo infrared fixed point $\left(\alpha_{*}\right)$, a la Caswell-Banks-Zack, based on the two-loop beta function in the large $N_{f} \mathrm{QCD} \stackrel{14}{ }$. During the walking region, the gauge coupling slowly reaches the critical coupling, which is slightly off from an infrared fixed point $\alpha_{*}$, $\alpha_{\mathrm{cr}}\left(<\alpha_{*}\right)$, where the chiral/electroweak symmetry is dynamically broken by technifermion condensation $\langle\bar{F} F\rangle \neq 0$ and hence technifermions get the dynamical mass $m_{F}$ on the order of $\mathrm{TeV}$, where $F_{\pi}$ denotes the technipion decay constant associated with the chiral symmetry breaking. The walking regime ends at two edges: below the infrared scale $m_{F} \sim \mathcal{O}(\mathrm{TeV})$, technifermions decouple and hence the balance with technigluon contributions gets lost, leading to the one edge of walking. The other edge is above the ultraviolet scale $\Lambda_{\mathrm{TC}}\left(\sim 10^{3}-10^{4} \mathrm{TeV}\right)$ the theory will be embedded into an extended technicolor (ETC) 15 .

The dynamical mass generation at $\alpha=\alpha_{\mathrm{cr}}$ is characterized by so-called Miransky scaling $\underline{16}$, tied with the conformal phase transition $\frac{17}{}$,

$$
m_{F} \sim \Lambda_{\mathrm{TC}} e^{-\frac{\pi}{\sqrt{\alpha / \alpha_{\mathrm{cr}}-1}}}, \quad \text { for } \quad \alpha>\alpha_{\mathrm{cr}} .
$$

This scaling property, i.e. the criticality actually supports the existence of the wide-range walking regime above $\alpha=\alpha_{\mathrm{cr}}$ in Fig. 1, i.e., the large scale hierarcy 

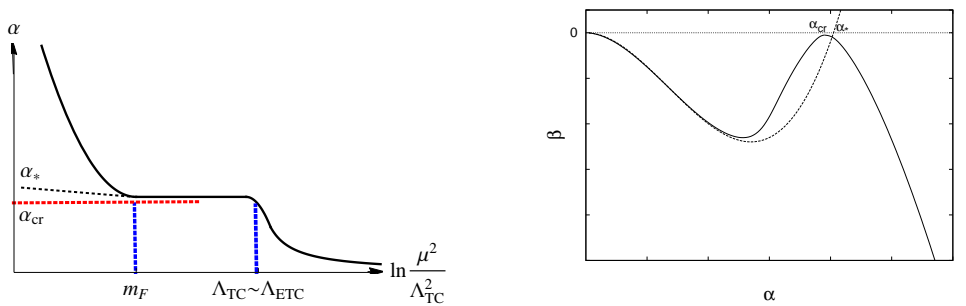

Fig. 1. A schematic picture of possible perturbative running coupling (left) and the beta function (right) in the region $\alpha<\alpha_{\mathrm{cr}}$, in comparison with the nonperturbative region $\alpha>\alpha_{\mathrm{cr}}$.

$m_{F} \ll \Lambda_{\mathrm{TC}}$. Thus, the WTC realizes a typical technihadron mass scale $M_{\mathrm{Had}}$ on the order of electroweak/ TeV scale, much smaller than $\Lambda_{\mathrm{TC}}$ :

$$
M_{\mathrm{Had}}=\mathcal{O}(\mathrm{EW}) \ll \Lambda_{\mathrm{TC}},
$$

in sharp contrast to QCD where $M_{\mathrm{Had}} \sim \Lambda_{\mathrm{QCD}}$.

As seen from Fig. 1, the WTC possesses the (approximate) scale invariance $\left(\beta(\alpha) \simeq 0\right.$ for $m_{F}<\mu<\Lambda_{\mathrm{TC}}$ ), which is spontaneously broken by the technifermion condensation/mass generation. This implies the presence of a (pseudo) NambuGoldstone boson ("dilaton") for the scale symmetry. which arises as a flavor-singlet composite scalar $\sim \bar{F} F$. This composite scalar is what we call the TD, technidilaton.

The mass of the TD is essentially provided by the "nonperturbative" scale anomaly of the WTC: according to the dynamical mass generation in Eq.(11), the gauge coupling $\alpha$ is renormalized, starts running and hence the "nonperturbative" beta function $\beta_{\mathrm{NP}}(\alpha)$ is generated $\frac{18}{}$ :

$$
\beta_{\mathrm{NP}}(\alpha)=\Lambda_{\mathrm{TC}} \frac{\partial \alpha}{\partial \Lambda_{\mathrm{TC}}}=-\frac{2 \alpha_{\mathrm{cr}}}{\pi}\left(\frac{\alpha}{\alpha_{\mathrm{cr}}}-1\right)^{3 / 2} .
$$

This induces the "nonperturbative" scale anomaly:

$$
\partial_{\mu} D^{\mu}=\frac{\beta_{\mathrm{NP}}(\alpha)}{4 \alpha^{2}}\left(\alpha G_{\mu \nu}^{2}\right) \neq 0,
$$

where $D_{\mu}$ denotes the dilatation current and $G_{\mu \nu}$ the field strength of technigluon field. Thus the TD becomes massive due to the nonperturbative scale anomaly ${ }_{4.5}$. Note that this scale anomaly is induced by the fermion mass generation itself: the scale symmetry is spontaneously broken by the technifermion condensation, at the same time it is explicitly broken by the nonperturbative running, as in Eq.(3), triggered by the technifermion mass generation itself.

The TD mass generation can be dictated by the partially conserved dilation current $(\mathrm{PCDC})$ relation,

$$
\left\langle 0\left|\theta_{\mu}^{\mu}\right| 0\right\rangle=\frac{F_{\phi}^{2} M_{\phi}^{2}}{4}
$$


where $\theta_{\mu}^{\mu}=\partial_{\mu} D^{\mu}$ is the trace of the energy-momentum tensor as in Eq. (44) and $F_{\phi}$ is the TD decay constant defined as

$$
\left\langle 0\left|D_{\mu}(x)\right| \phi(p)\right\rangle=-i p_{\mu} F_{\phi} e^{-i p x} .
$$

(The $F_{\pi}$ is not necessarily equal to $F_{\pi}$, can rather be larger, as it will turn out below. ) Since the scale anomaly is generated by the technifermion mass generation, one can find that the $\left\langle 0\left|\theta_{\mu}^{\mu}\right| 0\right\rangle$ generically scales like

$$
\left\langle 0\left|\theta_{\mu}^{\mu}\right| 0\right\rangle \sim N_{C} N_{F} m_{F}^{4},
$$

up to some loop factor dependent on details of nonperturbative computations. Combining this with the PCDC in Eq.(5D), one arrives at a generic scaling law of the TD mass,

$$
M_{\phi} \sim \sqrt{N_{C} N_{F}} \frac{m_{F}^{2}}{F_{\phi}} .
$$

There have been much progress of the WTC particularly on the light TD, not just in the ladder Schwinger-Dyson (SD) equation, but also in a variety of approaches such as the ladder Bethe-Salpeter equation combined with the ladder SD equation $\frac{19,20}{2}$, the effective theory based on the scale-invariant chiral perturbation theory $\stackrel{9.21}{ }$, holographic method $\underline{11,22,23}$, and eventually, the first-principle calculation of the flavor-singlet scalar meson in the large $N_{F}$ QCD on the lattice $24-27$. In particular, it is remarkable that such a light flavor-singlet scalar meson as a candidate for the TD was observed in the lattice $N_{F}=8 \mathrm{QCD} \underline{24}$, the theory shown to have signatures of the lattice walking theory including the mass anomalous dimension $\gamma_{m} \simeq 1 \underline{28} \underline{\underline{30}}$. Note that $N_{F}=8$ (four weak-doublets) corresponds to the "one-family model" $\underline{3,6}$ which is the most straightforward model building of the ETC $\underline{15}$ as a standard way to give masses to the quarks and leptons. The one-family model of the WTC with $N_{C}=4$ is in fact best fit to the $125 \mathrm{GeV}$ Higgs data $\underline{-11}$, which has been updated recently $\underline{\underline{13}}$ and will be summarized later, and is shown to be most natural for the ETC model building $\underline{31}$.

Below we shall propose a novel understanding for the realization of the light TD: it is the presence of the "anti-Veneziano limit", intrinsic to the WTC based on large $N_{F}$ QCD. This observation has separately been described in the recent paper $\underline{13}$.

\section{Parametrically Light Technidilaton in Large $N_{F}$ Walking Technicolor}

The key observation is that as long as the PCDC relation is satisfied, the TD as a pseudo NG boson has a vanishing mass in the anti-Veneziano limit, quite independently of the numerical details of nonperturbative calculations. To see this one should note from the large $N_{F}$ and $N_{C}$ scalings that

$$
m_{F} \sim \frac{F_{\pi}}{\sqrt{N_{C}}}, \quad F_{\phi} \sim \sqrt{N_{C} N_{F}} m_{F},
$$


and the $F_{\pi}$ is related to the electroweak scale $v_{\mathrm{EW}} \simeq 246 \mathrm{GeV}$ as $F_{\pi}=$ $1 / \sqrt{N_{F} / 2} v_{\text {EW }}$. Then one immediately finds that the TD mass scaling in Eq. (8) implies

$$
\frac{M_{\phi}}{v_{\mathrm{EW}}} \sim \frac{1}{\sqrt{N_{F} N_{C}}} .
$$

Now consider the anti-Veneziano limit where

$$
N_{C} \rightarrow \infty, \quad N_{F} \rightarrow \infty, \quad \text { with } \quad r \equiv \frac{N_{F}}{N_{C}} \gg 1 \quad \text { fixed } .
$$

In this limit one readily gets $M_{\phi} / v_{\mathrm{EW}} \rightarrow 0$ in the chiral/electroweak broken phase with $v_{\mathrm{EW}} \simeq 246 \mathrm{GeV}$ fixed. Thus the TD as the pseudo NG boson has a vanishing mass limit, though not exact massless point, in the anti-Veneziano limit, where the nonperturbative scale anomaly vanishes in units of $F_{\phi}$ via the PCDC in Eq. (5) as a measure of the spontaneous symmetry breaking of the scale symmetry. This is similar to the $\eta^{\prime}$ meson in QCD, which is regarded as a pseudo NG boson whose mass, evaluated through the anomalous WT identity with the $U(1)_{A}$ anomaly, does vanish in the large $N_{F}$ and $N_{C}$ limit with $r=N_{F} / N_{C}$ fixed $(\ll 1)$ (Veneziano limit): $M_{\eta^{\prime}}^{2} / F_{\pi}^{2} \sim N_{F} / N_{C}^{2} \rightarrow 0$, without the exact massless point. In this sense, the TD can be a vanishingly light pseudo NG boson at the same level as the $\eta^{\prime}$ in QCD.

Actually, more suprising thing will happen when one employs the ladder approximation for the WTC with the nonrunning gauge coupling. (The details has been given in the full paper ${ }^{13}$.) Then the fermion dynamical mass $m_{F}$ is related to the electroweak scale $v_{\mathrm{EW}}$ precisely through the Pagels-Stokar formula:

$$
v_{\mathrm{EW}}^{2}=(246 \mathrm{GeV})^{2} \simeq \frac{N_{F} N_{C}}{4 \pi^{2}} m_{F}^{2} \simeq m_{F}^{2}\left[\frac{N_{F}}{8} \frac{N_{C}}{4}\right],
$$

From this and Eq.(10) one can find a natural estimate of the TD mass for the one-family model with $N_{F}=8$ and $N_{C}=4$ to be

$$
M_{\phi}=\mathcal{O}\left(m_{F} / 2\right)=\mathcal{O}\left(v_{\mathrm{EW}} / 2\right)=\mathcal{O}(125 \mathrm{GeV}),
$$

in agreement with the LHC Higgs as the TD.

More precisely, one has

$$
M_{\phi}^{2} \simeq\left(\frac{v_{\mathrm{EW}}}{2}\right)^{2} \cdot\left(\frac{5 v_{\mathrm{EW}}}{F_{\phi}}\right)^{2} \cdot\left[\frac{8}{N_{F}} \frac{4}{N_{C}}\right] .
$$

Remarkablly, it was shown ${ }^{8-11}$ that the TD best fit to the current LHC data is realized when

$$
F_{\phi} \simeq 5 v_{\mathrm{EW}} \simeq 1.25 \mathrm{TeV} \quad \text { for } \quad M_{\phi}=125 \mathrm{GeV} \quad\left(N_{F}=8, N_{C}=4\right)
$$

(See also the later discussions). With the fact that $v_{\mathrm{EW}}^{2} \propto N_{F} N_{C} m_{F}^{2} \sim F_{\phi}^{2}$, the result reflects the generic scaling:

$$
\frac{M_{\phi}}{v_{\mathrm{EW}}} \sim \frac{M_{\phi}}{F_{\phi}} \sim \frac{m_{F}}{F_{\phi}} \sim \frac{1}{\sqrt{N_{F} N_{C}}} \rightarrow 0,
$$

in the anti-Veneziano limit. 


\section{The low-energy effective model for the TD}

The effective theory for the TD involving the SM particles are constructed based on the scale-invariant chiral perturbation theory ${ }^{9,21}$ with the scale anomaly terms supplied properly to the underlying WTC.

The chiral/electroweak and scale invariant Lagrangian thus takes the form ${ }^{9}$

$$
\mathcal{L}_{\mathrm{inv}}=\frac{v_{\mathrm{EW}}^{2}}{4} \chi^{2} \operatorname{tr}\left[D_{\mu} U^{\dagger} D^{\mu} U\right]+\mathcal{L}_{\mathrm{kin}}(\chi),
$$

where $\chi=e^{\phi / F_{\phi}}$ is a nonlinear base of the scale symmetry, which parametrizes the TD field $\phi$ with the decay constant $F_{\phi}$ and has the scale dimension $1 ; D_{\mu} U=\partial_{\mu} U-$ $i W_{\mu} U+i U B_{\mu}$ with the $S U(2)_{W}$ and $U(1)_{Y}$ gauge fields $W$ and $B ; \mathcal{L}_{\text {kin }}(\chi)$ denotes the scale invariant kinetic term of TD and $U$ the usual chiral field parameterizing the (eaten) Nambu-Goldstone boson fields $\pi$ as $U=e^{2 i \pi / v_{\mathrm{EW}}}$; The $\left|D_{\mu} U\right|^{2}$ term gives the TD couplings to massive weak bosons:

$$
g_{\phi W W / Z Z}=\frac{2 m_{W / Z}^{2}}{F_{\phi}} .
$$

As seen in Eqs. (3) and (4), the scale symmetry is actually broken explicitly as well as spontaneously by dynamical mass generation of technifermions, which has to be respected also in the nonlinear realization ${ }^{9}$. Such explicit breaking effects arise in the TD Yukawa couplings to the SM fermions, which reflect underlying ETCinduced four-fermion terms, and couplings to QCD gluons and photons related to the scale anomaly in the SM gauge sector. In order to incorporate these effects into the scale-invariant Lagrangian, we introduce a spurion field $S$ having the scale dimension 1 coupled to the SM fermions, digluon $g g$ and diphoton $\gamma \gamma$ in such a way that $\underline{\underline{9}}$

$$
\begin{aligned}
\mathcal{L}_{S}= & -m_{f}\left(\left(\frac{\chi}{S}\right)^{2-\gamma_{m}} \cdot \chi\right) \bar{f} f \\
& +\log \left(\frac{\chi}{S}\right)\left\{\frac{\beta_{F}\left(g_{s}\right)}{2 g_{s}} G_{\mu \nu}^{2}+\frac{\beta_{F}(e)}{2 e} F_{\mu \nu}^{2}\right\},
\end{aligned}
$$

where $G_{\mu \nu}$ and $F_{\mu \nu}$ respectively denote the field strengths for QCD gluon and photon fields; $g_{s}$ and $e$ are the QCD gauge and electromagnetic couplings, respectively; $\beta_{F} \mathrm{~s}$ are the beta functions only including the technifermion loop contributions.

The TD Yukawa coupling to the SM $f$-fermion arises from the first line of Eq. (19) as $\frac{5}{5}$

$$
g_{\phi f f}=\frac{\left(3-\gamma_{m}\right) m_{f}}{F_{\phi}},
$$

along with scale dimension of technifermion bilinear operator $\left(3-\gamma_{m}\right)$. The anomalous dimension $\gamma_{m} \simeq 1$ in WTC, which is crucial to obtain the realistic mass of the SM fermions of the first and the second generations without suffering from the flavor-changing neutral current problems. However it was known for long time that it is not enough for the mass of the third-generation SM $f$-fermions like $t, b, \tau$ : A 
simplest resolution would be the strong ETC model ${ }^{32}$ having much larger anomalous dimension $1<\gamma_{m}<2$ due to the strong effective four-fermion coupling from the ETC dynamics in addition to the walking gauge coupling. Here we take $\gamma_{m} \simeq$ 2, i.e., $\left(3-\gamma_{m}\right) \simeq 1$, as in the strong ETC model for the third-generation SM $f$-fermions like $t, b, \tau$ which are relevant to the current LHC data.

In addition, the TD potential should be included so as to reproduce the PCDC relation Eq. (5) in the underlying walking theory:

$$
V(\chi)=\frac{F_{\phi}^{2}}{4} m_{\phi}^{2} \chi^{4}\left(\log \frac{\chi}{S}-\frac{1}{4}\right) .
$$

Here we stress remark on stability of the light TD mass against radiative corrections. As a pseudo Nambu-Goldstone boson of scale invariance, the quadratic divergence is suppressed by the scale invariance for the walking regime $m_{F}\left(\simeq v_{\mathrm{EW}}\right)<$ $\mu<\Lambda_{\mathrm{TC}}\left(\sim \Lambda_{\mathrm{ETC}}\right)$. The scale symmetry breaking in the ultraviolet region $\mu>\Lambda_{\mathrm{TC}}$ has no problem thanks to the naturalness as usual just as in QCD where the theory has only logarithmic divergences. Only possible source of the scale symmetry violation is from an effective theory for $\mu<m_{F}\left(\simeq v_{\mathrm{EW}}\right)$. See Fig. 1

Now it turns out that the TD mass is stable against the feedback effects of the ETC through particularly the top quark loop, because of the large $F_{\phi} \simeq 5 v_{\mathrm{EW}}$ : below $\mu=m_{F}\left(\simeq v_{\mathrm{EW}}\right)$ the dominant corrections to the TD mass $M_{\phi}$ come from the SM top quark and TD self-loops. These can be estimated from the effective Lagrangian in Eqs.(17) and (19) including the SM sector and ETC effects, which are estimated to be 9,13

$$
\begin{aligned}
\frac{\left.\delta M_{\phi}^{2}\right|_{\phi^{4}}}{M_{\phi}^{2}} & \simeq 24 \frac{m_{F}^{2}}{\left(4 \pi F_{\phi}\right)^{2}} \simeq 6 \times 10^{-3} \\
\frac{\left.\delta M_{\phi}^{2}\right|_{\text {ETC } / \text { Yukawa }}}{M_{\phi}^{2}} & \simeq 12\left(3-\gamma_{m}\right)^{2} \frac{m_{F}^{2}}{\left(4 \pi F_{\phi}\right)^{2}} \frac{m_{t}^{2}}{M_{\phi}^{2}} \\
& \simeq\left(3-\gamma_{m}\right)^{2} \frac{\left.\delta M_{\phi}^{2}\right|_{\phi^{4}}}{M_{\phi}^{2}}
\end{aligned}
$$

where the cutoff has been set to $m_{F} \simeq v_{\mathrm{EW}}$. These yield

$$
\delta M_{\phi}^{2} / M_{\phi}^{2}=0.01\left(\gamma_{m}=2\right)-0.03\left(\gamma_{m}=1\right) .
$$

Thus the $125 \mathrm{GeV}$ TD mass is fully stable against the radiative corrections, in contrast to the unnatural SM Higgs case.

In passing, the TD potential in Eq. (21) written in terms $\chi=e^{\phi / F_{\phi}}$ is rewritten in the TD field $\phi$ as $\underline{9}$

$$
V(\phi)=-\mathcal{L}_{(2) \text { anomaly }}^{S}=-\frac{M_{\phi}^{2} F_{\phi}^{2}}{16}+\frac{1}{2} M_{\phi}^{2} \phi^{2}+\frac{4}{3} \frac{M_{\phi}^{2}}{F_{\phi}} \phi^{3}+2 \frac{M_{\phi}^{2}}{F_{\phi}^{2}} \phi^{4}+\cdots
$$

It is remarkable to notice that in the anti-Veneziano limit Eq. (11) the TD self 
couplings (trilinear and quartic couplings) are highly suppressed:

$$
\frac{4}{3} \frac{M_{\phi}^{2}}{F_{\phi}} \sim \frac{1}{\sqrt{N_{F} N_{C}}}, \quad 2 \frac{M_{\phi}^{2}}{F_{\phi}^{2}} \sim \frac{1}{N_{F} N_{C}}
$$

by $M_{\phi} / F_{\phi} \sim 1 / \sqrt{N_{F} N_{C}}$ and $M_{\phi} \sim N_{F}^{0} N_{C}^{0}$. It is also interesting to numerically compare the TD self couplings for the one-family model $\left(N_{F}=8, N_{C}=4\right)$ having $v_{\text {EW }} / F_{\phi} \simeq 1 / 5$ with the self couplings of the SM Higgs with $m_{h}=M_{\phi}$, by making the ratios:

$$
\begin{gathered}
\left.\frac{g_{\phi^{3}}}{g_{h_{\mathrm{SM}}^{3}}}\right|_{M_{\phi}=m_{h}}=\left.\frac{\frac{4 M_{\phi}^{2}}{3 F_{\phi}}}{\frac{m_{h}^{2}}{2 v_{\mathrm{EW}}}}\right|_{M_{\phi}=m_{h}} \simeq \frac{8}{3}\left(\frac{v_{\mathrm{EW}}}{F_{\phi}}\right) \simeq 0.5, \\
\left.\frac{g_{\phi^{4}}}{g_{h_{\mathrm{SM}}^{4}}}\right|_{M_{\phi}=m_{h}}=\left.\frac{\frac{2 M_{\phi}^{2}}{F_{\phi}^{2}}}{\frac{m_{h}^{2}}{8 v_{\mathrm{EW}}^{2}}}\right|_{M_{\phi}=m_{h}}=16\left(\frac{v_{\mathrm{EW}}}{F_{\phi}}\right)^{2} \simeq 0.6 .
\end{gathered}
$$

This shows that the TD self couplings, although generated by the strongly coupled interactions, are even smaller than those of the SM Higgs, a salient feature of the approximate scale symmetry in the ant-Veneziano limit. This is in sharp contrast to the widely-believed folklore, "Strong coupling solutions like Technicolor tend to lead to a strongly coupled Higgs" $\underline{33}$.

\section{LHC Higgs vs. Technidilaton in One-Family Model with $N_{C}=4$ and $N_{F}=8$}

One finds from the effective Lagrangian that the TD couplings to the SM gauge bosons and the SM fermions can just be obtained by scaling from the SM Higgs as $v_{\mathrm{EW}} \rightarrow F_{\phi} \underline{\underline{\underline{9}} \cdot 10}$ :

$$
\begin{aligned}
\frac{g_{\phi W W / Z Z}}{g_{h_{\mathrm{SM}} W W / Z Z}} & =\frac{g_{\phi f f}}{g_{h_{\mathrm{SM}} f f}} \quad(\text { for } \quad f=t, b, \tau) \\
& =\frac{v_{\mathrm{EW}}}{F_{\phi}} \quad\left[\simeq \frac{1}{5} \ll 1 \quad\left(N_{F}=8, N_{C}=4\right)\right] .
\end{aligned}
$$

On the other hand, in the one-family model with $N_{F}=8$ the couplings to digluon and diphoton include the colored/charged techni-fermion loop contributions along with a factor $N_{C} \underline{9}, 10$,

$$
\begin{array}{r}
\mathcal{L}_{\mathrm{eff}}^{\gamma \gamma, g g}=\frac{\phi}{F_{\phi}}\left\{\frac{\beta_{F}\left(g_{s}\right)}{2 g_{s}} G_{\mu \nu}^{2}+\frac{\beta_{F}(e)}{e} F_{\mu \nu}^{2}\right\}, \\
\beta_{F}\left(g_{s}\right)=\frac{g_{s}^{3}}{(4 \pi)^{2}} \frac{4}{3} N_{C}, \quad \beta_{F}(e)=\frac{e^{3}}{(4 \pi)^{2}} \frac{16}{9} N_{C},
\end{array}
$$


where the beta functions have been evaluated at the one-loop level. Thus one finds the scaling from the SM Higgs $\frac{10}{}$,

$$
\begin{aligned}
\frac{g_{\phi g g}}{g_{h_{\mathrm{SM}} g g}} \simeq \frac{v_{\mathrm{EW}}}{F_{\phi}} \cdot\left(1+2 N_{C}\right), \\
\frac{g_{\phi \gamma \gamma}}{g_{h_{\mathrm{SM} \gamma \gamma}}} \simeq \frac{v_{\mathrm{EW}}}{F_{\phi}} \cdot\left(\frac{63-16}{47}-\frac{32}{47} N_{C}\right),
\end{aligned}
$$

where in estimating the SM contributions we have incorporated only the top (the terms of 1 and 16/47 for $g g$ and $\gamma \gamma$ rates, respectively) and the $W$ boson (the term of $63 / 47$ for $\gamma \gamma$ rate) loop contributions.

The scaling laws for the TD couplings to the SM fermions and weak bosons in Eq.(27) are similar to those for the couplings of other dilatons, or radions 34 , while the TD couplings to diphoton and digluon in Eq.(29) are significantly differrent in a sense that these couplings include contributions beyond the SM (technifermion loop contributions), in contrast to other types of dilatons/radions.

In Table 1 the branching fractions for relevant decay channels of the TD at 125 $\mathrm{GeV}$ are listed in the case of the one-family model with $N_{C}=4$. Note that the total width $\Gamma_{\text {tot }}=1.15 \mathrm{MeV}$ is smaller than the SM Higgs, which reflects the weaker couplings than the those of the latter, in contrast to the widely spread folklore.

Table 1. The TD branching ratios at $125 \mathrm{GeV}$ in the one-family model with $N_{C}=4$. The total width is also given.

\begin{tabular}{|c|c|c|c|c|c|c|c|c|}
\hline BR[\%] & $g g$ & $b b$ & $W W$ & $Z Z$ & $\tau \tau$ & $\gamma \gamma$ & $Z \gamma$ & $\mu \mu$ \\
\hline \hline$\Gamma_{\text {tot }}=1.15 \mathrm{MeV}$ & 75.1 & 19.6 & 3.56 & 0.38 & 1.19 & 0.068 & 0.0048 & 0.0042 \\
\hline
\end{tabular}

Calculating the signal strengths for the LHC production categories (gluon gluon fusion $(\mathrm{ggF})$, vector boson fusion $(\mathrm{VBF})$, vector boson associate production $(\mathrm{VH})$ and top associate production $(\mathrm{ttH}))$,

$$
\mu_{X_{1} X_{2}}^{i}=\frac{\sigma_{\phi}^{i} \times \mathrm{BR}\left(\phi \rightarrow X_{1} X_{2}\right)}{\sigma_{\mathrm{h}_{\mathrm{SM}}}^{i} \times \mathrm{BR}\left(h \rightarrow X_{1} X_{2}\right)},
$$

as a function of the overall coupling $v_{\mathrm{EW}} / F_{\phi}$ for given the number of $N_{C}$, we may fit the $\mu_{X_{1} X_{2}}^{i}$ to the latest data on the Higgs coupling measurements ${ }^{35}$. to determine the best-fit value of $v_{\mathrm{EW}} / F_{\phi}$. The result of the goodness of fit is shown in Table 2 , which updates the previous analysis 10 . The Table 2 shows that the TD in the onefamily model with $N_{C}=4$ is favored by the current LHC Higgs data as much the same level as the SM Higgs. Remarkably, the best fit value $\left[v_{\mathrm{EW}} / F_{\phi}\right]_{\text {best }} \simeq 0.2$, i.e. $F_{\phi} \simeq 5 v_{\mathrm{EW}}$ for $N_{C}=4$ is in excellent agreement with the ladder estimate of the $\mathrm{TD}$ mass $\simeq 125 \mathrm{GeV}$ in Eq.(15)!

In Table 3 we also make a list of the predicted signal strengths for each production category for the best fit value of $v_{\mathrm{EW}} / F_{\phi} \simeq 0.23$ in the case with $N_{C}=4$, along with the latest result reported from the ATLAS and CMS experiments 35 . Note the TD signal strengths in the dijet category (VBF), which involves the contamination 
by about $30 \%$ from the ggF + gluon jets, $g g \rightarrow \phi+g g$. The contribution from the ggF is highly enhanced compared to the SM Higgs case, due to the extra techniquark loop contribution, which compensates the overall suppression by the direct VBF coupling $v_{\mathrm{EW}} / F_{\phi} \simeq 0.2$ to lift the event rate up to be comparable to the SM

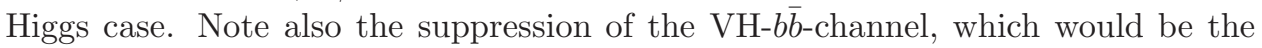
characteristic signature of the TD to be distinguishable from the SM Higgs. More data from the upcoming LHC Run-II will draw a conclusive answer to whether or not the LHC Higgs is the SM Higgs, or the TD.

Table 2. The best fit values of $v_{\mathrm{EW}} / F_{\phi}$ for the one-family model with $N_{C}=3,4,5$ displayed together with the minimum of the $\chi^{2}\left(\chi_{\min }^{2}\right)$ normalized by the degree of freedom. Also has been shown in the last column the case of the SM Higgs corresponding to $N_{C}=0$ and $v_{\mathrm{EW}} / F_{\phi}=1$.

\begin{tabular}{|c|c|c|}
\hline$N_{C}$ & {$\left[v_{\mathrm{EW}} / F_{\phi}\right]_{\text {best }}$} & $\chi_{\min }^{2} /$ d.o.f. \\
\hline \hline 3 & 0.27 & $25 / 17 \simeq 1.5$ \\
\hline 4 & 0.23 & $16 / 17 \simeq 0.92$ \\
\hline 5 & 0.17 & $32 / 17 \simeq 2.0$ \\
\hline \hline 0 [SM Higgs] & 1 & $8.0 / 18 \simeq 0.44$ \\
\hline
\end{tabular}

Table 3. The predicted signal strengths of the TD with $v_{\mathrm{EW}} / F_{\phi}=0.23$ in the case of the one-family model with $N_{C}=4$. The numbers in the parentheses correspond to the amount estimated without contamination from the ggF process. Also have been displayed the latest data on the Higgs coupling measurements reported from the ATLAS and CMS experiments 35 .

\begin{tabular}{|c|c|c|}
\hline TD signal strengths $\left(v_{\mathrm{EW}} / F_{\phi}=0.23, N_{C}=4\right)$ & ATLAS & CMS \\
\hline \hline$\mu_{\gamma \gamma}^{\mathrm{ggF}} \simeq 1.4$ & $1.32 \pm 0.38$ & $1.13 \pm 0.35$ \\
\hline$\mu_{Z Z}^{\mathrm{ggF}} \simeq 1.0$ & $1.7 \pm 0.5$ & $0.83 \pm 0.28$ \\
\hline $\mathrm{a} \mu_{W W}^{\mathrm{ggF}} \simeq 1.0$ & $0.98 \pm 0.28$ & $0.72 \pm 0.37$ \\
\hline$\mu_{\tau \tau}^{\mathrm{ggF}} \simeq 1.0$ & $2.0 \pm 1.4$ & $1.1 \pm 0.46$ \\
\hline \hline$\mu_{\gamma \gamma}^{\mathrm{BF}} \simeq 0.87(0.019)$ & $0.8 \pm 0.7$ & $1.16 \pm 0.59$ \\
\hline$\mu_{Z Z}^{\mathrm{BF}} \simeq 0.61(0.014)$ & $0.3 \pm 1.3$ & $1.45 \pm 0.76$ \\
\hline$\mu_{W W}^{\mathrm{BF}} \simeq 0.61(0.014)$ & $1.28 \pm 0.51$ & $0.62 \pm 0.53$ \\
\hline$\mu_{\tau \tau}^{V \mathrm{BF}} \simeq 0.61(0.014)$ & $1.24 \pm 0.57$ & $0.94 \pm 0.41$ \\
\hline$\mu_{b b}^{\mathrm{VH}} \simeq 0.014$ & $0.52 \pm 0.40$ & $1.0 \pm 0.50$ \\
\hline
\end{tabular}

The ATLAS and CMS have made a plot of the LHC Higgs couplings to the SM particles against the SM particle masses $\frac{36}{6}$, shown that the LHC Higgs couplings to fermions have aligned very well with the SM Higgs boson properties. The plot has been made by assuming no contributions beyond the SM in loops, i.e., no contributions beyond SM to diphoton and digluon couplings. However, as explicitly seen from Eq.(29), the technidilaton couplings to diphoton and digluon significantly include the terms beyond the SM, technifermion contributions charged under the $U(1)_{\mathrm{em}}$ or $\mathrm{QCD}$ color. In this respect, such a plot cannot be applied 
to the technidilaton. In fact, the successful consistency with the LHC Higgs coupling measurement, as shown in Table 3 is due to those beyond SM contributions, which especially enhance the ggF production cross section, balanced by the overall suppression due to the coupling $F_{\phi}$ larger than $v_{\mathrm{EW}}$ by a factor of 5 .

\section{Other Walking Technihadrons: Technipions and Technirhos}

In addition to the TD, the WTC predicts the rich spectrum. Such typical technihadrons involve the technipions, technirhos (and techni $a_{1} \mathrm{~s}$.) In the case of the one-family model having the chiral $S U(8)_{L} \times S U(8)_{R}$ symmetry broken down to the vectorial $S U(8)_{V}$, the technipion spectra can be classified by the SM gauge charges, as well as the technirhos and techni- $a_{1} \mathrm{~s}$.

\subsection{Walking Technipions}

Technipion masses are all from explicit breaking outside of the WTC sector, i.e, SM gauge interactions and ETC gauge interactions. One can estimate the technipion masses in the WTC, based on the first order perturbation of the explicit chiral symmetry breaking by the "weak gauge couplings" of SM gauge interactions and the ETC gauge interactions (Dashen's formula). This is the same strategy as the QCD estimate of the $\pi^{+}-\pi^{0}$ mass difference. It turns out $\underline{23,37,38}$ that the technipion masses are enhanced through the chiral condensate by the anomalous dimension as $\left(Z_{m}^{-1}\right)^{2} \sim\left(\Lambda / m_{F}\right)^{2 \gamma_{m}} \underline{4.39,40}$, to be on the order of TeV scale:

$$
M_{\pi} \sim \mathcal{O}(\mathrm{TeV}) .
$$

It is striking that although the explicit chiral symmetry breakings are formally very small due to the "weak gauge couplings", the nonperturbative contributions from the WTC sector lift all the technipions masses to the TeV region so that they all lose the nature of the "pseudo NG bosons". This is actually a universal feature of the dynamics with large anomalous dimension, "amplification of the symmetry violation" $\underline{41}$. This amplification effect should not be confused with that of the pseudo NG boson mass due to the technifermion bare mass effects, like the pion mass due to the current quark mass, $F_{\pi}^{2} m_{\pi}^{2}=2 m\langle\bar{\psi} \psi\rangle$, which are not amplified by the large anomalous dimension, since the mass operator times the mass is totally invariant against the renormalization. (In the actual technicolor model, all the technifermions are set to be exactly massless and such a type of explicit breaking is not considered anyway.)

Note that although the left-over light spectra are just three exact NG bosons absorbed into $W / Z$ bosons, our theory with $N_{F} \gg 2$ in the anti-Veneziano limit is completely different from the model with massless flavors $N_{f}=2$ where the symmetry breaking is $S U(2)_{L} \times S U(2)_{R} / S U(2)_{V}$. In fact, even though all the NG bosons, other than the three exact NG bosons to be absorbed into $W, Z$ bosons, are massive and decoupled from the low energy physics, they are composite of the linear combinations of all the $N_{F}$ technifermions, not just 2 of them. 
Including the walking technipions one can discuss the LHC phenomenology based on the scale-invariant chiral perturbation theory described by the Lagrangian in Eq.(17) 38. The LHC-Run I limits on the 60 massive technipions were evaluated to be 23,38

$$
\begin{array}{lll}
\text { color-octet technipions }(\theta): & M_{\theta}>1.5-1.6 \mathrm{TeV} & \text { from } \theta \rightarrow t \bar{t} \\
\text { color-triplet technipions }(T): & M_{T}>1.0-1.1 \mathrm{TeV} & \text { from leptoquark search } . \\
\text { color-singlet technipions }(P): & M_{P}>850 \mathrm{GeV} & \text { from } \theta \rightarrow t \bar{t}
\end{array}
$$

More data from the Run II will thus reveal if walking technipions with the mass as large as $\mathcal{O}(\mathrm{TeV})$ are present.

\subsection{Non-pseudo NG-technihadrons: walking technirhos}

In contrast to the TD and technipions, all the non-NG boson technihadrons, such as the technirho, techni- $a_{1}$, etc., have no constraints from the PCDC as the explicit breaking of the scale symmetry but do have constraints from the spontaneous breaking of the scale symmetry, so that they should have masses on the scale of spontaneous breaking of the scale symmetry, characterized by $F_{\phi}$ much larger than $2 m_{F}$ of the naive nonrelativistic quark model picture:

$$
M_{\rho}, M_{a_{1}}, \cdots=\mathcal{O}\left(\mathrm{TeV}^{\prime} \mathrm{s}\right)>\mathcal{O}\left(F_{\phi}\right) \gg 2 m_{F} \gg M_{\phi} .
$$

In fact, the infrared conformal physics of the WTC should be described by the low-lying composite fields as effective fields, in a way to realize all the symmetry structure of the underlying theory.

Such an effective theory of WTC is constructed as a straightforward extension of scale-invariant chiral perturbation theory ${ }_{9.21}$, i.e, the scale-invariant version 42 of the Hidden Local Symmetry (HLS) model ${ }^{43,44}$, (the "s-HLS model"), where the technirho mass terms have the scale-invariance nonlinearly realized by the TD field $\chi=e^{\phi / F_{\phi}}$, with the spontaneous breaking of the scale invariance characterized by the scale of $F_{\phi}$, while the Higgs (TD) mass term in the TD potential, on the order of $m_{F}\left(\ll F_{\phi}\right)$, is the only source of the explicit breaking of the scale symmetry related (via PCDC) to the nonperturbative scale anomaly of the underlying theory.

One interesting candidate for such technihadrons may be a resonance behind the diboson excess recently observed at the $\mathrm{LHC}$ at $2 \mathrm{TeV} \stackrel{45,46}{ }$, which can be identified with the walking technirho $\stackrel{47}{ }$. The excesses suggest a characteristically small width $\Gamma_{\text {total }}<100 \mathrm{GeV}^{45}$, which can be naturally realized in the anti-Veneziano limit in Eq.(11):

$$
\frac{\Gamma_{\text {total }}}{M_{\rho}} \simeq \frac{\Gamma(\rho \rightarrow W W / W Z)}{M_{\rho}} \simeq \frac{1}{48 \pi} \frac{g_{\rho \pi \pi}^{2}}{N_{D}} \sim \frac{1}{N_{F} N_{C}} \rightarrow 0
$$

where $N_{D}=N_{F} / 2$ is the number of the weak-doublets. In fact our one-family model $N_{F}=8, N_{C}=4$ can reproduces the features of the excesses very well $\stackrel{47}{ }$. A smoking gun of the walking technirho is the absence of the decay to the $125 \mathrm{GeV}$ Higgs (TD), which is forbidden by the scale symmetry explicitly broken only by 
the Higgs (TD) mass term (corresponding to the nonperturbative scale anomaly in the underlying WTC)르. Actually, the salient feature of the scale symmetry of the generic effective theory containing the SM gauge bosons and the Higgs plus new vector bosons (any other massive particles as well), is the absence of the decay of the new vector bosons such as the technirho (and also other higher resonances) into the $125 \mathrm{GeV}$ Higgs plus the SM gauge bosons $\underline{48}$. If such decays of new particles are not found at LHC Run II, then the $125 \mathrm{GeV}$ Higgs is nothing but the dilaton (TD in the case of the WTC) responsible for the nonlinearly realized scale symmetry, i.e., the spontaneous breaking of the scale symmetry, no matter what underlying theory may be beyond the SM. This should be tested in the ongoing LHC Run-II.

\section{Conclusion}

The technidilaton, arising as a pseudo NG boson for spontaneous breaking of the scale symmetry in the walking techniicolor, can be identified with the Higgs discovered at the LHC. The smallness of the TD mass can be ensured by the characteristic feature of the walking, and the presence of the anti-Veneziano limit in the case of the large-flavor walking gauge theory including the one-family model. The couplings of the technidilaton to the standard model particles have so far been consistent with those of the LHC Higgs. Crucial deviation from the standard model Higgs will be found in the decay channel to bottom quark pair, which is significantly suppressed by the large decay constant compared to the electroweak scale, to be tested in the ongoing Run II or other future colliders.

Walking technipions, pseudo NG bosons for the chiral symmetry, get heavy due to the salient feature of the walking dynamics having the large anomalous dimension. The masses are lifted to be on the order of a few TeV scale, which is consistent with the current LHC-Run I limits, so the walking technipions may be uncovered soon, or more severely constrained at the ongoing Run II through each discovery channel.

Non-pseudo technihadrons in the walking technicolor cannot be so light, in contrast to the technidilaton. The masses are thus expected to be on the order of the scale of the scale symmetry breaking, TeV scale, slightly higher than the electro weak scale. Among those Non-pseudos, the walking technirhos at around $2 \mathrm{TeV}$ may be responsible for the recent excesses in the diboson channel, reported from the ATLAS group. The ongoing Run II will give the definite answer if it is the signal of the walking technirho.

\section{Acknowledgments}

I would like to thank Hidenori S. Fukano, Masafumi Kurachi and Koichi Yamawaki for useful discussions and collaborations. This work was supported by the JSPS Grant-in-Aid for Scientific Research (S) \#22224003 and the JSPS Grant-in-Aid for Young Scientists (B) \#15K17645. 


\section{References}

1. G. Aad et al. [ATLAS Collaboration], Phys. Lett. B 716, 1 (2012); S. Chatrchyan et al. [CMS Collaboration], Phys. Lett. B 716, 30 (2012).

2. S. Weinberg, Phys. Rev. D 13, 974 (1976); L. Susskind, Phys. Rev. D 20, 2619 (1979).

3. For a review see E. Farhi and L. Susskind, Phys. Rept. 74, 277 (1981).

4. K. Yamawaki, M. Bando and K. Matumoto, Phys. Rev. Lett. 56, 1335 (1986); M. Bando, T. Morozumi, H. So and K. Yamawaki, Phys. Rev. Lett. 59, 389 (1987).

5. M. Bando, K. Matumoto and K. Yamawaki, Phys. Lett. B 178, 308 (1986).

6. S. Dimopoulos, Nucl. Phys. B 168, 69 (1980).

7. S. Matsuzaki and K. Yamawaki, Prog. Theor. Phys. 127, 209 (2012);

8. Phys. Rev. D 85, 095020 (2012).

9. S. Matsuzaki and K. Yamawaki, Phys. Rev. D 86, 035025 (2012).

10. Phys. Lett. B 719, 378 (2013).

11. S. Matsuzaki and K. Yamawaki, Phys. Rev. D 86, 115004 (2012).

12. S. Matsuzaki, arXiv:1304.4882 [hep-ph].

13. S. Matsuzaki and K. Yamawaki, arXiv:1508.07688 [hep-ph].

14. W. E. Caswell, Phys. Rev. Lett. 33, 244 (1974); T. Banks and A. Zaks Nucl. Phys. B 196, 189 (1982).

15. S. Dimopoulos and L. Susskind, Nucl. Phys. B 155, 237 (1979); E. Eichten and K. D. Lane, Phys. Lett. B 90, 125 (1980).

16. V. A. Miransky, Nuovo Cim. A 90, 149 (1985).

17. V. A. Miransky and K. Yamawaki, Phys. Rev. D 55, 5051 (1997); Errata, 56, 3768 (1997).

18. W. A. Bardeen, C. N. Leung and S. T. Love, Phys. Rev. Lett. 56, 1230 (1986); Nucl. Phys. B 273, 649 (1986).

19. M. Harada, M. Kurachi and K. Yamawaki, Phys. Rev. D 68, 076001 (2003).

20. M. Kurachi and R. Shrock, JHEP 0612, 034 (2006).

21. S. Matsuzaki and K. Yamawaki, Phys. Rev. Lett. 113, 082002 (2014).

22. K. Haba, S. Matsuzaki and K. Yamawaki, Phys. Rev. D 82, 055007 (2010).

23. M. Kurachi, S. Matsuzaki and K. Yamawaki, Phys. Rev. D 90, no. 9, 095013 (2014).

24. Y. Aoki, T. Aoyama, M. Kurachi, T. Maskawa, K. Miura, K. -i. Nagai, H. Ohki and E. Rinaldi, A. Shibata, K. Yamawaki and T. Yamazaki (the LatKMI Collaboration), Phys. Rev. D 89, 111502(R) (2014); PoS LATTICE 2013 (2013) 070 .

25. Y. Aoki, T. Aoyama, M. Kurachi, T. Maskawa, K. -i. Nagai, H. Ohki and E. Rinaldi, A. Shibata, K. Yamawaki and T. Yamazaki (the LatKMI Collaboration), "Light composite scalar in twelve-flavor QCD on the lattice," Phys. Rev. Lett. 111, no. 16, 162001 (2013).

26. Z. Fodor, K. Holland, J. Kuti, D. Nogradi and C. H. Wong, PoS LATTICE 
2013, 062 (2014) arXiv:1401.2176 [hep-lat]].

27. R. Brower, A. Hasenfratz, C. Rebbi, E. Weinberg and O. Witzel, arXiv:1411.3243 [hep-lat].

28. Y. Aoki, T. Aoyama, M. Kurachi, T. Maskawa, K. -i. Nagai, H. Ohki, A. Shibata, K. Yamawaki and T. Yamazaki (The LatKMI Collaboration), Phys. Rev. D 87, 094511 (2013).

29. T. Appelquist et al. [LSD Collaboration], Phys. Rev. D 90, 114502 (2014).

30. A. Hasenfratz, D. Schaich and A. Veernala, arXiv:1410.5886 [hep-lat].

31. M. Kurachi, R. Shrock and K. Yamawaki, Phys. Rev. D 91, no. 5, 055032 (2015).

32. V. A. Miransky, K. Yamawaki, Mod. Phys. Lett. A4, 129-135 (1989); K. Matumoto, Prog. Theor. Phys. 81, 277-279 (1989); T. Appelquist, M. Einhorn, T. Takeuchi, L. C. R. Wijewardhana, Phys. Lett. B220, 223 (1989).

33. see, e.g., Talk given at Aspen Winter, https://indico.cern.ch/event/202554/session/3/material/slides/0?contribId=58

34. W. D. Goldberger, B. Grinstein and W. Skiba, Phys. Rev. Lett. 100, 111802 (2008); J. Fan, W. D. Goldberger, A. Ross and W. Skiba, Phys. Rev. D 79, 035017 (2009); L. Vecchi, Phys. Rev. D 82, 076009 (2010); L. Randall and R. Sundrum, Phys. Rev. Lett. 83, 3370 (1999); 83, 4690 (1999); W. D. Goldberger and M. B. Wise, Phys. Rev. Lett. 83, 4922 (1999); B. Coleppa, T. Gregoire and H. E. Logan, Phys. Rev. D 85, 055001 (2012); V. Barger, M. Ishida and W. Y. Keung, Phys. Rev. D 85, 015024 (2012); Phys. Rev. Lett. 108, 101802 (2012); B. A. Campbell, J. Ellis and K. A. Olive, JHEP 1203, 026 (2012); V. Barger and M. Ishida, Phys. Lett. B 709, 185 (2012).

35. The ATLAS collaboration, ATLAS-CONF-2015-007, ATLAS-COM-CONF2015-011; ATLAS-CONF-2013-012, ATLAS-COM-CONF-2013-015; G. Aad et al. [ATLAS Collaboration], Phys. Lett. B 726, 88 (2013) [Erratum-ibid. B 734, 406 (2014)]; ATLAS-CONF-2013-030, ATLAS-COM-CONF-2013-028; ATLAS-CONF-2013-108, ATLAS-COM-CONF-2013-095; ATLAS-CONF-2013079, ATLAS-COM-CONF-2013-080; CMS-PAS-HIG-13-001; CMS-PAS-HIG-13002; S. Chatrchyan et al. [CMS Collaboration], JHEP 1401, 096 (2014); CMS Collaboration [CMS Collaboration], CMS-PAS-HIG-13-022; S. Chatrchyan et al. [CMS Collaboration], JHEP 1405, 104 (2014); S. Chatrchyan et al. [CMS Collaboration], Phys. Rev. D 89, no. 1, 012003 (2014).

36. The ATLAS and CMS Collaborations, ATLAS-CONF-2015-044.

37. M. Harada, M. Kurachi and K. Yamawaki, Prog. Theor. Phys. 115, 765 (2006).

38. J. Jia, S. Matsuzaki and K. Yamawaki, Phys. Rev. D 87, 016006 (2013).

39. B. Holdom, Phys. Rev. D 24, 1441 (1981).

40. K. Yamawaki and T. Yokota, Phys. Lett. B 113, 293 (1982); Nucl. Phys. B 223, 144 (1983).

41. For reviews, see, e.g., K. Yamawaki, Lecture at 14th Symposium on Theoret- 
ical Physics, Cheju, Korea, July 1995, arXiv:hep-ph/9603293 C. T. Hill and E. H. Simmons, Phys. Rept. 381, 235 (2003) [Erratum-ibid. 390, 553 (2004)]; F. Sannino, Acta Phys. Polon. B40, 3533-3743 (2009); K. Yamawaki, in Proceedings of Moriond EW Conference 2013, arXiv:1305.6352 [hep-ph].

42. M. Kurachi, S. Matsuzaki and K. Yamawaki, Phys. Rev. D 90, 055028 (2014).

43. M. Bando, T. Kugo, S. Uehara, K. Yamawaki, and T. Yanagida, Phys. Rev. Lett. 54 (1985) 1215; M. Bando, T. Kugo, and K. Yamawaki, Nucl. Phys. B259 (1985) 493; M. Bando, T. Fujiwara, and K. Yamawaki, Prog. Theor. Phys. 79 (1988) 1140; M. Bando, T. Kugo, and K. Yamawaki, Phys. Rept. 164 (1988) $217-314$.

44. M. Harada and K. Yamawaki, Phys. Rept. 381 (2003) 1-233.

45. G. Aad et al. [ATLAS Collaboration], arXiv:1506.00962 [hep-ex].

46. V. Khachatryan et al. [CMS Collaboration], JHEP 1408, 173 (2014).

47. H. S. Fukano, M. Kurachi, S. Matsuzaki, K. Terashi and K. Yamawaki, Phys. Lett. B 750, 259 (2015).

48. H. S. Fukano, S. Matsuzaki and K. Yamawaki, arXiv:1507.03428 [hep-ph]. 